\title{
VARIABILIDADE ESPACIAL DE ATRIBUTOS FÍSICOS DE UM ARGISSOLO VERMELHO-AMARELO SOB PASTAGEM E VEGETAÇÃO SECUNDÁRIA EM REGENERAÇÃO NATURAL
}

\author{
JULIÃO S. DE S. LIMA ${ }^{1}$, MARCOS A. SATTLER ${ }^{2}$, RENATO R. PASSOS ${ }^{3}$, \\ PAULO C. OLIVEIRA II ${ }^{4}$, GUSTAVO S. DE SOUZA ${ }^{5}$
}

\begin{abstract}
RESUMO: Este trabalho foi realizado com o objetivo de estudar a variabilidade espacial de atributos físicos de um Argissolo Vermelho-Amarelo sob pastagem e vegetação secundária em regeneração natural. Duas áreas foram escolhidas em uma encosta, com a amostragem de solo à profundidade de 0-0,2 $\mathrm{m}$, com os pontos georreferenciados em uma malha regular de 10x10 m, totalizando 64 pontos em cada área. Foram avaliados o volume total de poros, macroporosidade, microporosidade, densidade do solo, resistência do solo à penetração e umidade do solo em cada ponto. Os atributos estudados na área de pastagem apresentam indicador de compactação do solo pelo pisoteio de animais, com estrutura moderada e forte dependência espacial, com exceção da macroporosidade e a resistência à penetração. $\mathrm{Na}$ área de vegetação secundária $(\mathrm{VN})$, somente a macroporosidade não apresenta dependência espacial. $\mathrm{O}$ volume total de poros e a densidade do solo apresentam o mesmo padrão espacial na área sob pastagem.
\end{abstract}

PALAVRAS - CHAVE: manejo do solo, geoestatística, semivariograma.

\section{SPATIAL VARIABILITY OF PHYSICAL ATTRIBUTES OF A RED-YELLOW ULTISOL UNDER PASTURE AND SECONDARY VEGETATION IN NATURAL REGENERATION}

\begin{abstract}
This work was carried out with the objective of studying the spatial variability of the physical attributes of a Red-Yellow Ultisol under pasture and secondary vegetation in natural regeneration. Two areas were chosen in a hillside, with the soil sampling to the depth of $0-0.2 \mathrm{~m}$, with the georeferenced points in a regular grid of $10 \times 10 \mathrm{~m}$, totalizing 64 points. In each point it was evaluated the total volume of porosity, macroporosity, microporosity, bulk density, soil penetration resistance and soil water content. The studied attributes in the pasture area present indicator of soil compaction for the animals' traffic, with moderate and strong structure of spatial dependence, except for the macroporosity and penetration resistance. In the area of secondary vegetation (VN) only the macroporosity does not present spatial dependence. The total volume of porosity and the bulk density present the same spatial standard in the area under pasture.
\end{abstract}

KEYWORDS: soil management, geostatistics, semivariogram.

\section{INTRODUÇÃO}

A Bacia Hidrográfica do Itapemirim situa-se na região sul do Estado do Espírito Santo (ES) e possui a maior parte de sua área ocupada por pastagem (com pecuária de leite e, mais recentemente, com pecuária de corte). Juntamente com a cultura do café, essas atividades constituem a base de sua economia. Na medida em que o uso e a ocupação da área ocorrem sem critérios técnicos, percebe-se crescente degradação dos recursos naturais, refletindo-se na baixa capacidade produtiva dos solos, associada a outros danos ambientais também de significativa importância (SATTLER, 2006).

Historicamente, a alteração de ecossistemas naturais, por meio da retirada da cobertura vegetal para fins de implantação de culturas, tem promovido o rompimento do equilíbrio entre o

\footnotetext{
${ }^{1}$ Eng ${ }^{\circ}$ Agrícola, Prof. Associado, Departamento de Engenharia Rural, UFES, Alegre - ES, limajss@ yahoo.com.br

${ }^{2}$ Eng ${ }^{\circ}$ Agrônomo, M.Sc., Escola Agrotécnica Federal de Alegre - EAFA, Alegre - ES.

${ }^{3}$ Eng ${ }^{\mathrm{o}}$ Agrônomo, Prof. Adjunto, Departamento de Produção Vegetal, UFES, Alegre - ES.

${ }^{4}$ Eng ${ }^{\circ}$ Mecânico, Prof. Adjunto, Departamento de Engenharia Rural, UFES, Alegre - ES.

${ }^{5}$ Eng ${ }^{0}$ Agrônomo, Doutorando em Engenharia Agrícola - UNICAMP, Campinas - SP.

Recebido pelo Conselho Editorial em: 21-6-2007
}

Aprovado pelo Conselho Editorial em: 17-4-2009 
solo e o meio, modificando seus atributos químicos, físicos e biológicos, limitando sua utilização sustentável nos processos produtivos e tornando-o mais suscetível à erosão.

A caracterização da variabilidade espacial dos atributos do solo, associada a outras técnicas de tomada de decisão, e a experiência do agricultor são importantes para o refinamento das práticas de manejo e a avaliação dos efeitos da agricultura sobre a qualidade ambiental.

O estudo da dependência espacial de atributos do solo, por meio da teoria das variáveis regionalizadas ou geoestatística, permite a interpretação e a projeção dos resultados com base na estrutura da sua variabilidade natural, podendo indicar alternativas de uso, além de possibilitar melhor compreensão da variabilidade dos atributos e sua influência sobre a produção das culturas (OLIVEIRA, 2007).

Para o entendimento sobre os atributos no processo de degradação do solo por ocupação diferenciada na região, desenvolveu-se este trabalho com o objetivo de analisar a variabilidade espacial dos atributos físicos de um Argissolo Vermelho-Amarelo sob pastagem e vegetação secundária em regeneração natural.

\section{MATERIAL E MÉTODOS}

A área de estudo localiza-se na Bacia Hidrográfica do Itapemirim, sub-bacia do Córrego Horizonte, na Escola Agrotécnica Federal de Alegre, situada no distrito de Rive, Município de Alegre, a sudoeste do Estado do Espírito Santo e pertence à interface entre os ambientes "Cachoeiro" e "Celina", segundo LANI (1987).

O local escolhido para a instalação de duas malhas regulares de 80 x $80 \mathrm{~m}$, com pontos distantes de $10 \mathrm{~m}$, situa-se na face sudeste de uma encosta, sendo escolhido o terço médio da rampa com declividade entre 30 e 45 graus, conforme modelo digital de elevação (MDE) (Figura 1). As amostras de solo foram coletadas na camada de 0-0,20 m, em solo classificado como Argissolo Vermelho-Amarelo com textura argilosa (EMBRAPA, 1999).

As coordenadas geográficas da área que compõem as duas malhas, considerando os quatro

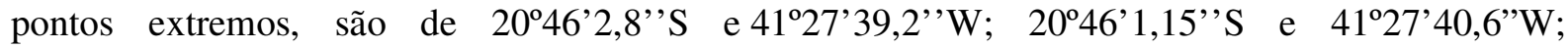
$20^{\circ} 45^{\prime} 59,6$ 'S e $41^{\circ} 27^{\prime} 30,0^{\prime \prime} \mathrm{W} ; 2^{\circ} 45^{\prime} 57,3^{\prime \prime}$ S e $41^{\circ} 27^{\prime} 35,8^{\prime \prime} \mathrm{W}$.

A área de pastagem (PA) está cultivada com braquiária (Brachiaria decumbens), com aproximadamente seis anos de implantação pela aração com tração animal, em nível e correção da acidez do solo na época de plantio. O sistema de pastejo é extensivo, com média anual de 1,0 UA ha ${ }^{-1}$, sendo maior concentração observada nos períodos de chuva, podendo chegar a 3,0 UA ha ${ }^{-1}$, e menor concentração (ou retirada dos animais da área) nos períodos mais secos. A segunda cobertura vegetal compreende vegetação secundária em regeneração natural (VN) há aproximadamente 30 anos. Os principais representantes do extrato arbóreo são: angico-canjiquinha (Peltophorium dubium); ipê-felpudo (Zeyhera tuberculosa); jacaré (Piptadenia communis); cinco-folhas (Sparattosperma vernicosum), extrato arbustivo: arranha-gato (Acacia spp.) e, nas mais abertas, incidência de capim-colonião (Panicum maximum Jacq.).

Na caracterização física do solo, segundo EMBRAPA (1997), a densidade do solo (Ds) foi determinada com amostrador Uhland, utilizando-se de amostras indeformadas retiradas em anel de aço de bordas cortantes; a umidade (U), pelo método termogravimétrico, em estufa a $105^{\circ} \mathrm{C}$; a resistência do solo à penetração (RP) foi obtida com a utilização de penetrômetro de impacto (STOLF, 1991); a microporosidade (MiP) no aparelho extrator de Richards submetido à tensão de $0,006 \mathrm{MPa}$; o volume total de poros (VTP) calculado pela equação: VTP $=[1-(\mathrm{Ds} / \mathrm{Dp})] 100$, em que $\mathrm{Dp}=\mathrm{a}$ densidade de partículas do solo, determinada pelo método do balão volumétrico, com valor médio de $2,65 \mathrm{~kg} \mathrm{dm}^{-3}$, e a macroporosidade $(\mathrm{MaP})$, pela diferença entre VTP e MiP. 


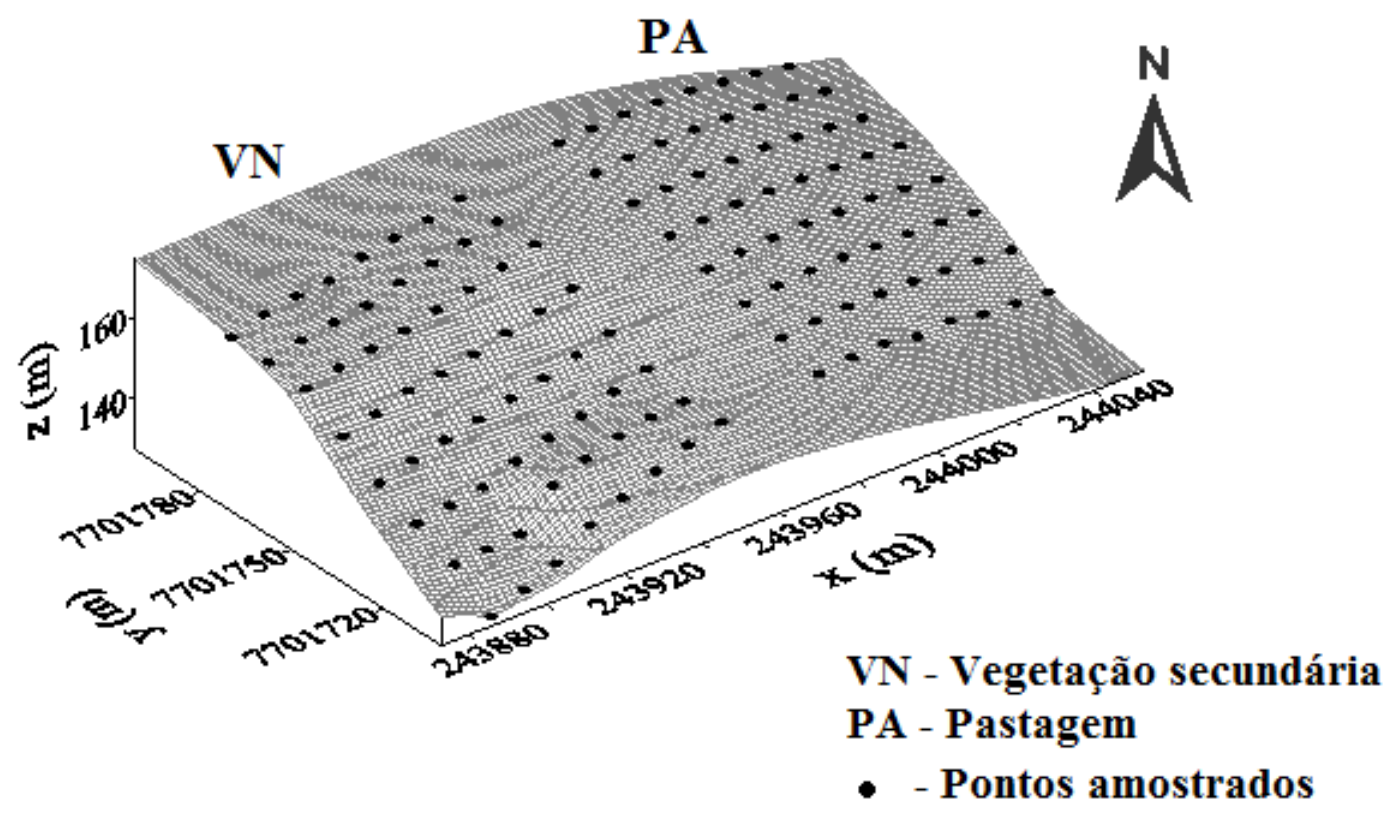

FIGURA 1. Representação topográfica da área experimental com os pontos amostrados na pastagem (PA) e vegetação secundária em regeneração natural (VN). Topographic representation of the experimental area with the sampled points in the pasture (PA) and secondary vegetation in natural regeneration (VN).

Os atributos físicos do solo, nas duas áreas, foram analisados por meio da análise estatística descritiva com medidas de posição e dispersão e uma análise exploratória dos dados, bem como o teste Shapiro-Wilks, a 5\% de probabilidade, para testar a normalidade dos dados. A geoestatística foi utilizada para verificar a existência e, nesse caso, quantificar o grau de dependência espacial dos valores dos atributos físicos, a partir do ajuste de funções teóricas aos modelos de semivariogramas experimentais, segundo VIEIRA et al. (1983), com base na pressuposição de estacionaridade da hipótese intrínseca, a qual é estimada pela eq. (1):

$$
\gamma^{*}(h)=\frac{1}{2 N(h)} \sum_{i=1}^{N(h)}\left[Z\left(x_{i}\right)-Z\left(x_{i}+h\right)\right]^{2}
$$

em que,

$\mathrm{N}(\mathrm{h})$ - número de pares experimentais de observações $\mathrm{Z}(\mathrm{xi}), \mathrm{Z}(\mathrm{xi}+\mathrm{h})$, separados por um vetor $h$.

Nos ajustes dos modelos teóricos aos semivariogramas, determinaram-se os parâmetros efeito pepita $\left(\mathrm{C}_{0}\right)$, patamar $\left(\mathrm{C}_{0}+\mathrm{C}_{1}\right)$, variância estrutural $\left(\mathrm{C}_{1}\right)$ e alcance (a). Os modelos considerados foram ajustados pelo software $\mathrm{GS}^{+}$(ROBERTSON, 1998), conforme descrito por SILVA et al. (2007). Para a determinação do índice de dependência espacial (IDE), utilizou-se da relação $\left[\mathrm{C}_{1} /\left(\mathrm{C}_{0}+\mathrm{C}_{1}\right)\right]$, conforme critérios estabelecidos por ZIMBACK (2001), que classifica o IDE $\leq 0,25$, de fraco; $0,25<$ IDE $<0,75$, de moderado e IDE $>0,75$, de forte.

Para a seleção do modelo teórico que melhor ajusta as semivariâncias experimentais, foram observados o maior $\mathrm{R}^{2}$ (coeficiente de determinação) e a menor SQR (soma de quadrados dos resíduos) (GUIMARÃES, 2000).

Em seguida, os semivariogramas foram escalonados pela variância dos dados, de acordo com VIEIRA (2000), para facilitar as interpretações e as comparações entre os semivariogramas de diferentes atributos. Conhecendo-se o semivariograma de cada atributo e havendo dependência 
espacial entre as amostras, realizou-se interpolação pelo método da krigagem ordinária para estimar valores em locais não medidos.

\section{RESULTADOS E DISCUSSÃO}

Os resultados da estatística descritiva para os atributos do solo sob pastagem (PA) e vegetação secundária, em regeneração natural (VN), estão na Tabela 1. Verifica-se que os atributos MiP, MaP, Ds e U, na área de PA, e MiP, Ds, RP e U, na área de VN, apresentam distribuição com assimetria negativa. Nesses casos, observa-se que a mediana é maior que a média, mostrando tendência para a concentração de valores maiores que essa. Com exceção da RP, na área de pastagem, e da MiP, na área de $\mathrm{VN}$, os demais atributos apresentam distribuição normal pelo teste Shapiro-Wilk's, embora a não normalidade seja comum quando se trata de dados obtidos na natureza (WEBSTER, 1985).

TABELA 1. Estatística descritiva dos atributos físicos do solo nas áreas sob pastagem (PA) e vegetação secundária em regeneração natural (VN). Descriptive statistics of soil physical attributes in areas under pasture (PA) and secondary vegetation in natural regeneration $(\mathrm{VN})$.

\begin{tabular}{|c|c|c|c|c|c|c|c|c|c|c|}
\hline \multirow{2}{*}{ Atributo } & \multirow{2}{*}{ Unidade } & \multirow{2}{*}{ Média } & \multirow{2}{*}{ Md. } & \multirow[t]{2}{*}{$S$} & \multirow{2}{*}{$\frac{\text { Valores }}{\text { Mín. }}$} & \multicolumn{3}{|c|}{ Coeficientes } & \multicolumn{2}{|c|}{ DN } \\
\hline & & & & & & Máx. & C.V. & $\mathrm{C}_{\mathrm{k}}$ & $\mathrm{C}_{\mathrm{s}}$ & $p$ valor \\
\hline \multicolumn{11}{|l|}{ Pastagem } \\
\hline VTP & $\left(\mathrm{m}^{3} \mathrm{~m}^{-3}\right)$ & 0,408 & 0,408 & 0,025 & 0,343 & 0,466 & 6,22 & $-0,01$ & 0,09 & $0,66^{*}$ \\
\hline $\mathrm{MiP}$ & $\left(\mathrm{m}^{3} \mathrm{~m}^{-3}\right)$ & 0,333 & 0,338 & 0,018 & 0,291 & 0,378 & 5,55 & $-0,30$ & $-0,18$ & $0,26^{*}$ \\
\hline $\mathrm{MaP}$ & $\left(\mathrm{m}^{3} \mathrm{~m}^{-3}\right)$ & 0,073 & 0,075 & 0,182 & 0,033 & 0,114 & 28,49 & $-0,43$ & $-0,04$ & $0,71 *$ \\
\hline Ds & $\left(\mathrm{kg} \mathrm{dm}^{-3}\right)$ & 1,56 & 1,56 & 0,07 & 1,41 & 1,73 & 4,30 & $-0,04$ & $-0,09$ & $0,66^{*}$ \\
\hline $\mathrm{RP}$ & $(\mathrm{MPa})$ & 5,95 & 5,76 & 0,86 & 3,80 & 7,39 & 14,4 & $-0,45$ & 0,22 & 0,04 \\
\hline $\mathrm{U}$ & $\left(\mathrm{kg} \mathrm{kg}^{-1}\right)$ & 0,177 & 0,179 & 0,012 & 0,144 & 0,206 & 7,00 & 0,02 & $-0,06$ & $0,84^{*}$ \\
\hline \multicolumn{11}{|c|}{ Vegetação secundária em regeneração natural } \\
\hline VTP & $\left(\mathrm{m}^{3} \mathrm{~m}^{-3}\right)$ & 0,464 & 0,464 & 0,027 & 0,409 & 0,539 & 5,74 & 0,15 & 0,43 & $0,53 *$ \\
\hline MiP & $\left(\mathrm{m}^{3} \mathrm{~m}^{-3}\right)$ & 0,332 & 0,336 & 0,019 & 0,270 & 0,374 & 5,85 & 0,86 & $-0,87$ & 0,013 \\
\hline $\mathrm{MaP}$ & $\left(\mathrm{m}^{3} \mathrm{~m}^{-3}\right)$ & 0,132 & 0,129 & 0,029 & 0,072 & 0,208 & 19,97 & 0,02 & 0,37 & $0,60 *$ \\
\hline Ds & $\left(\mathrm{kg} \mathrm{dm}^{-3}\right)$ & 1,43 & 1,43 & 0,06 & 1,29 & 1,57 & 4,52 & $-0,34$ & $-0,16$ & $0,80 *$ \\
\hline $\mathrm{RP}$ & $(\mathrm{MPa})$ & 4,03 & 4,13 & 0,83 & 2,17 & 6,09 & 22,32 & $-0,06$ & $-0,37$ & $0,15^{*}$ \\
\hline $\mathrm{U}$ & $\left(\mathrm{kg} \mathrm{kg}^{-1}\right)$ & 0,183 & 0,184 & 0,014 & 0,147 & 0,222 & 7,53 & 0,62 & $-0,23$ & $0,42 *$ \\
\hline
\end{tabular}

Atrib. - atributos; Md. - mediana; S - desvio-padrão; Mín. - valor mínimo; Máx. - valor máximo; Coeficientes: variação (C.V.); $\mathrm{C}_{\mathrm{k}}$ - curtose; $\mathrm{C}_{\mathrm{s}}$ - assimetria; * - distribuição normal; VTP - volume total de poros; MiP - microporosidade; MaP - macroporosidade; Ds - densidade do solo; RP - resistência à penetração, e U - umidade do solo.

A Ds foi o atributo que apresentou os menores coeficientes de variação (C.V.), com valores de $4,30 \%$ e 4,52\% para PA e VN, respectivamente. Entretanto, a MaP apresentou o maior C.V. $(28,49 \%)$ na PA, e a RP $(22,32 \%)$, na VN. O C.V. dos atributos VTP, MiP e DS está na mesma faixa de valores encontrados por SOUZA et al. (2004), em solo cultivado com cana-de-açúcar, e AZEVEDO (2004), em estudo com áreas de pastagem e vegetação natural. Adotando o critério de classificação para o C.V., proposto por WARRICK \& NIELSEN (1980), os mesmos revelaram-se: baixos (C.V. < 12\%) para VTP, MiP, DS e U, e médios $(12<$ C.V. $<60 \%)$ para MaP e RP, nas duas áreas.

$\mathrm{Na}$ área sob PA, os valores médios encontrados para os atributos VTP, MiP e Ds foram de $0,408 \mathrm{~m}^{3} \mathrm{~m}^{-3} ; 0,333 \mathrm{~m}^{3} \mathrm{~m}^{-3}$ e $1,56 \mathrm{~kg} \mathrm{dm}^{-3}$, respectivamente. Valores médios para VTP na ordem de $0,380 \mathrm{~m}^{3} \mathrm{~m}^{-3}$; MiP de $0,250 \mathrm{~m}^{3} \mathrm{~m}^{-3}$ e Ds de $1,54 \mathrm{~kg} \mathrm{dm}^{-3}$ foram detectados por SOUZA \& ALVES (2003), em pastagem em solo de cerrado, na camada de 0-0,20 m, no Mato Grosso do Sul. Segundo esses mesmos autores, essas diferenças podem ocorrer devido a alguns fatores na formação do solo e manejo intenso adotado em área plana, com presença de animais pesados e em grande número. Segundo AZEVEDO (2004), deve-se observar que o número de animais em áreas de pastagem 
tende a aumentar nas estações chuvosas em razão da maior disponibilidade de forragem, provocando efeito nos atributos do solo pelo pisoteio com umidade inadequada. Os maiores valores de VTP encontrados na área sob VN podem estar associados às melhores condições estruturais do solo sob essa cobertura vegetal em relação à área sob PA. ALBUQUERQUE et al. (2001) encontraram valores superiores para esse atributo em solo sob floresta subtropical, comparativamente a diversos tratamentos sob ação antrópica.

$\mathrm{O}$ valor médio da densidade do solo (Ds), determinado na área sob $\mathrm{VN}$, foi de $1,43 \mathrm{~kg} \mathrm{dm}^{-3}$, próximo de $1,46 \mathrm{~kg} \mathrm{dm}^{-3}$ encontrado por MAGALHÃES et al. (2001) e bem acima dos valores de $1,10 \mathrm{~kg} \mathrm{dm}^{-3}$ e $1,20 \mathrm{~kg} \mathrm{dm}^{-3}$ determinados por SÁ et al. (2003) e AZEVEDO (2004), respectivamente. $\mathrm{O}$ valor elevado de Ds encontrado na área de $\mathrm{VN}$ pode estar relacionado ao tempo de repouso da área para regeneração, lembrando que, anteriormente, a mesma também foi utilizada como pastagem.

De acordo com ARCHER \& SMITH (1972), o limite máximo tolerado da Ds para Argissolo é de $1,20 \mathrm{~kg} \mathrm{dm}^{-3}$, sendo que solos com Ds acima de $1,30 \mathrm{~kg} \mathrm{dm}^{-3}$ apresentam sérias desvantagens quanto à permeabilidade e aeração. SPERA (1995) e ALVARENGA (1996) encontraram valores de Ds inferiores em solos sob vegetação natural, comparados a outros tipos de cobertura. JORGE (1985) obteve resultados semelhantes e atribuiu os maiores valores para Ds a fatores pedogenéticos e/ou compactação oriunda do manejo, como no caso do excessivo pisoteio de bovinos. MAGALHÃES et al. (2001) afirmam que a tendência no aumento da Ds pode ser creditada ao tempo de pastejo e à degradação da própria pastagem.

$\mathrm{Na}$ área de PA, analisando a MaP de $0,073\left(\mathrm{~m}^{3} \mathrm{~m}^{-3}\right)$ e a RP no solo de 5,95 MPa, verifica-se, conforme TORMENA (2002) e AZEVEDO (2004), a presença de compactação na camada de 0-0,20 m. IMHOFF et al. (2001) propõem o limite aceitável para o atributo RP em torno de 2 e $3 \mathrm{MPa}$. Segundo ALVARENGA et al. (1996) e TORMENA (2002), valor de macroporosidade menor que $0,100\left(\mathrm{~m}^{3} \mathrm{~m}^{-3}\right)$ imprime inadequada difusão de oxigênio para atender às necessidades do sistema radicular e às atividades dos microrganismos no solo. Entretanto, no solo sob VN, os valores de MaP foram superiores $\left(0,132 \mathrm{~m}^{3} \mathrm{~m}^{-3}\right)$, indicando melhor agregação do solo. $\mathrm{O}$ maior valor de RP foi observado no solo da área sob PA, acompanhado de elevados valores de Ds e menores teores de água do solo (U), conforme também relatado por KLEIN et al. (1998) e TORRES \& SARAIVA (1999).

Os dados da análise da variabilidade espacial, por meio de semivariogramas escalonados, para os atributos do solo nas áreas PA e VN, com os parâmetros e modelos ajustados aos semivariogramas, são apresentados na Tabela 2. Observa-se que os atributos MaP e RP, na área sob $\mathrm{PA}$, e MaP, na área sob VN, apresentam efeito pepita puro (EPP), ou seja, ausência de dependência espacial para distâncias maiores que a menor distância de amostragem, que foi de $10 \mathrm{~m}$, distribuindo-se de forma independente no espaço.

O modelo linear (LIN) ajustou-se para U no solo sob PA, indicando tendência dos dados na área ou que a densidade de pontos amostrados não foi suficiente para estabilizar o patamar e, com isso, satisfazer a hipótese intrínseca. A remoção de tendência pelos métodos da mediana e dos polinômios não produziu efeito significativo nos modelos de semivariogramas dessas variáveis. Nesses casos, optou-se pela não utilização da modelagem dos resíduos.

O modelo esférico (ESF) ajustou-se para MiP, na área sob PA, e para os atributos VTP, MiP, RP e U, na área de VN. Para VTP e Ds, na área sob PA, e Ds na de VN, o ajuste foi com o modelo exponencial (EXP). GUIMARÃES (2000) encontrou ajuste do modelo ESF para VTP em solo cultivado sob sistema de plantio direto no cerrado em malha amostral com as mesmas dimensões usadas neste estudo. Isso indica que a variabilidade espacial de determinado atributo físico não depende só dos fatores de formação do solo, mas também do manejo adotado, conforme enfatiza CASTRIGNANO et al. (2000). 
TABELA 2. Dados dos semivariogramas escalonados para os atributos físicos nas áreas de pastagem (PA) e vegetação secundária em regeneração natural (VN). Semivariograms standardized data for the physical attributes in the areas of pasture (PA) and secondary vegetation in natural regeneration (VN).

\begin{tabular}{cccccccc}
\hline Atributos & Modelo & $\mathrm{a}(\mathrm{m})$ & $\mathrm{C}_{0}+\mathrm{C}_{1}$ & $\mathrm{C}_{0}$ & $\mathrm{R}^{2}$ & IDE & Classe Espacial \\
\hline VTP & EXP & 30,5 & 0,86 & 0,17 & 0,40 & 0,80 & Alta \\
MiP & ESF & 38,3 & 0,92 & 0,25 & 0,83 & 0,73 & Moderada \\
MaP & EPP & - & - & 0,98 & - & - & - \\
Ds & EXP & 29,8 & 0,85 & 0,16 & 0,41 & 0,80 & Alta \\
RP & EPP & - & - & 0,95 & - & - & - \\
U & LIN & - & 1,41 & 0,40 & 0,93 & 0,71 & Moderada \\
\hline \multicolumn{7}{c}{ Vegetação secundária em regeneração natural } & \\
VTP & ESF & 19,2 & 1,00 & 0,20 & 0,60 & 0,80 & Alta \\
MiP & ESF & 46,0 & 0,84 & 0,34 & 0,90 & 0,60 & Moderada \\
MaP & EPP & - & - & 1,00 & & - & - \\
Ds & EXP & 25,0 & 0,91 & 0,22 & 0,42 & 0,76 & Alta \\
RP & ESF & 42,5 & 1,13 & 0,18 & 0,84 & 0,84 & Alta \\
U & ESF & 23,0 & 1,00 & 0,12 & 0,51 & 0,88 & Alta \\
\hline
\end{tabular}

ESF - modelo esférico; EXP - modelo exponencial; EPP - efeito pepita puro; LIN - modelo linear sem patamar; a alcance; $\mathrm{C}_{0}+\mathrm{C}_{1}$ - patamar; $\mathrm{C}_{0}$ - efeito pepita; IDE - índice de dependência espacial, e $\mathrm{R}^{2}$ - coeficiente de determinação múltipla.
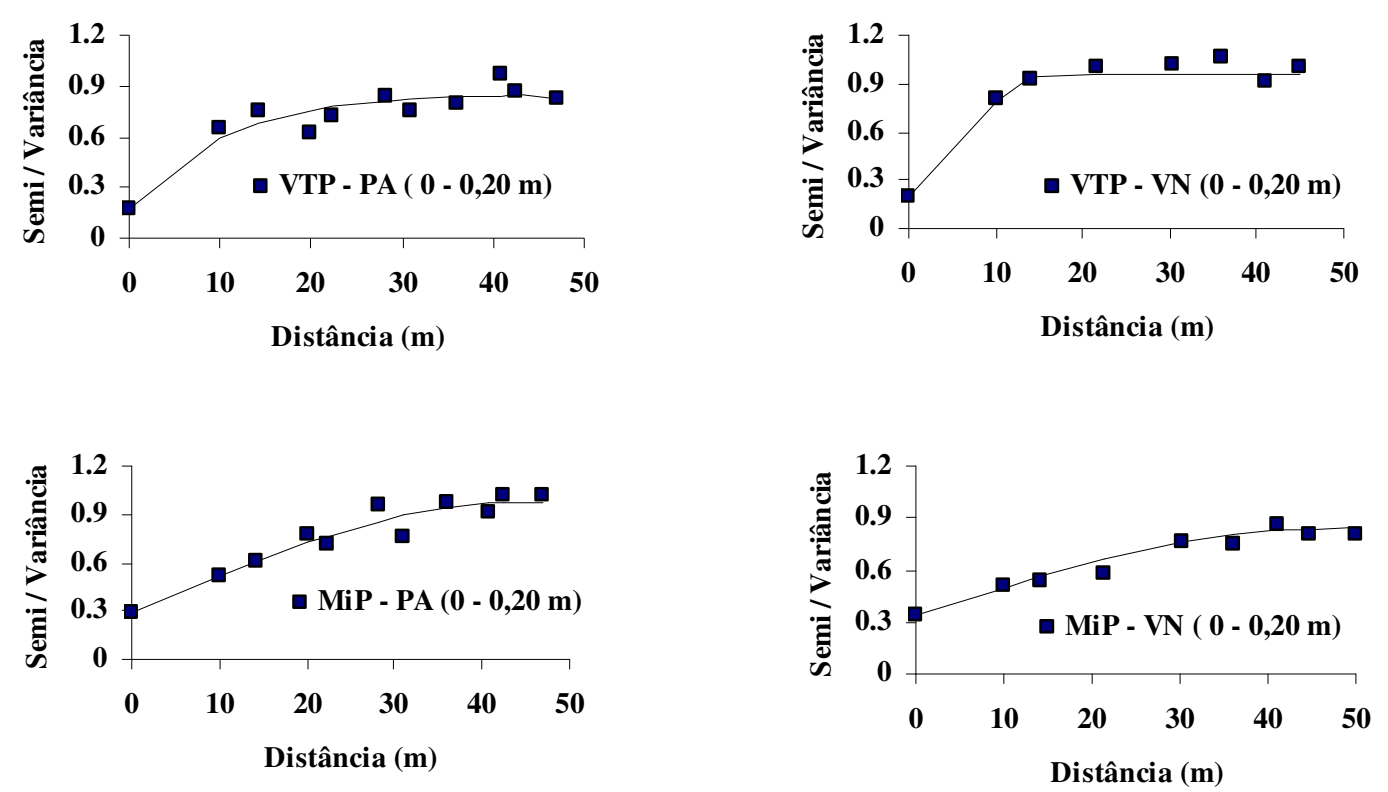

FIGURA 2. Semivariogramas escalonados dos atributos volume total de poros - VTP $\left(\mathrm{m}^{3} \mathrm{~m}^{-3}\right)$, microporosidade - MiP $\left(\mathrm{m}^{3} \mathrm{~m}^{-3}\right)$ e macroporosidade - $\mathrm{MaP}\left(\mathrm{m}^{3} \mathrm{~m}^{-3}\right)$, das áreas de pastagem (PA) e vegetação secundária em regeneração natural (VN). Standardized semivariograms of the total volume of pores attributes - VTP $\left(\mathrm{m}^{3} \mathrm{~m}^{-3}\right)$, microporosity - MiP $\left(\mathrm{m}^{3} \mathrm{~m}^{-3}\right)$ and macroporosity - MaP $\left(\mathrm{m}^{3} \mathrm{~m}^{-3}\right)$, areas of pasture (PA) and secondary vegetation in natural regeneration (VN). 

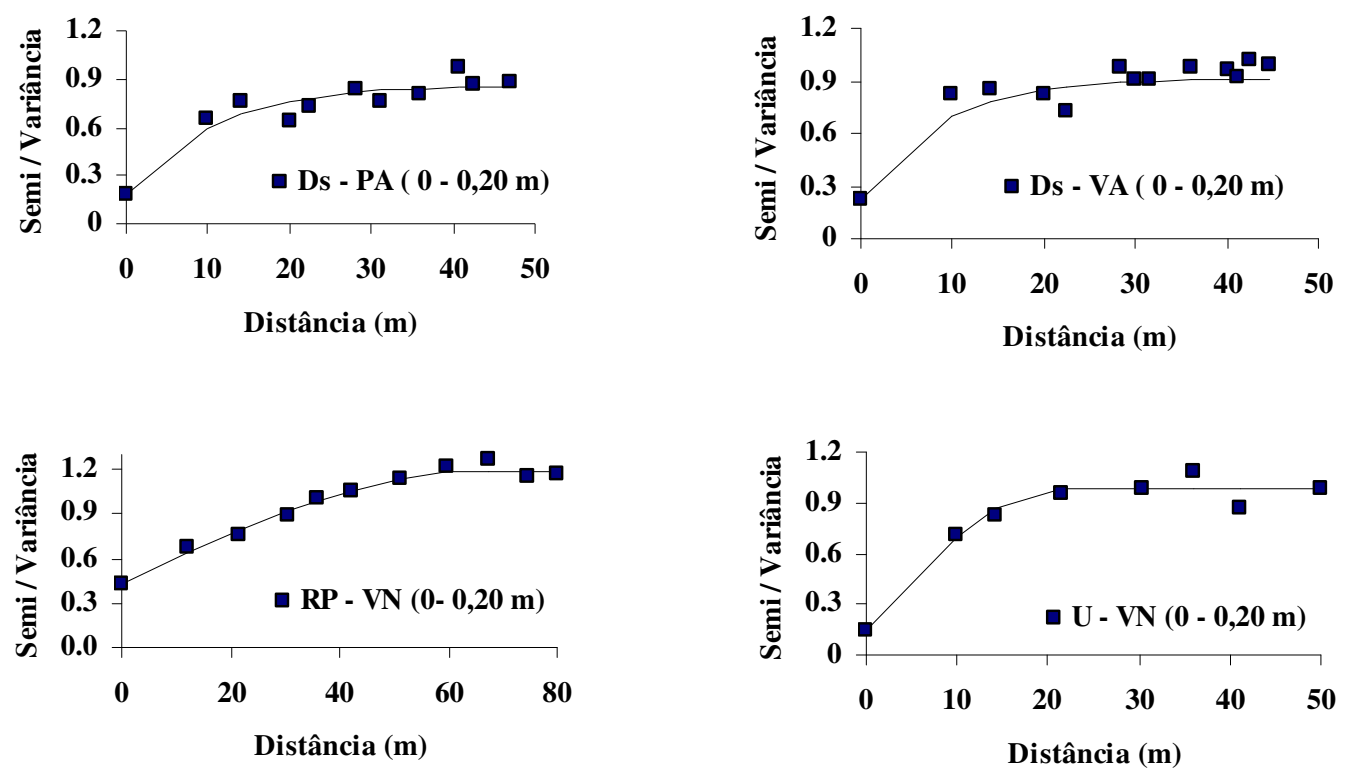

FIGURA 3. Semivariogramas escalonados dos atributos densidade do solo - Ds $\left(\mathrm{kg} \mathrm{dm}^{-3}\right)$, resistência do solo à penetração - RP $(\mathrm{MPa})$, e umidade do solo - $\mathrm{U}\left(\mathrm{kg} \mathrm{kg}^{-1}\right)$, das áreas de pastagem (PA) e vegetação secundária em regeneração natural (VN). Standardized semivariograms of the bulk density attributes - Ds $\left(\mathrm{kg} \mathrm{dm}^{-3)}\right.$, resistance of the soil to the penetration - RP (MPa) and soil moisture $\mathrm{U}\left(\mathrm{kg} \mathrm{kg}^{-1}\right)$, areas of pasture (PA) and secondary vegetation in natural regeneration $(\mathrm{VN})$.

O maior alcance obtido foi de $46 \mathrm{~m}$ para o atributo MiP, no solo sob VN, indicando maior continuidade espacial. Já na área sob PA, os atributos VTP e Ds apresentam o mesmo padrão de dependência espacial com os mesmos modelos de ajuste, efeito pepita, patamar e alcances de 30,5 e 29,8 m, respectivamente, confirmando, assim, a relação direta existente entre esses atributos.

Os semivariogramas escalonados dos atributos físicos do solo nas áreas de PA e VN estão apresentados nas Figuras 2 e 3. Para os atributos estudados, o índice de dependência espacial (IDE) apresentou valores altos $(>0,75)$ para VTP e Ds, no solo sob PA, e para os atributos VPT, Ds, RP e U, no solo sob VN. A MiP e a U no solo com PA e a MiP para a VN apresentam IDE moderado (entre 0,25 a 0,75 ).

Com relação aos valores de $\mathrm{R}^{2}$ para os semivariogramas escalonados, na área de PA, dois atributos (VTP e Ds) apresentam valores inferiores e dois atributos (MiP e U) superiores a 0,50. No solo sob VN, com exceção da MiP, todos os atributos apresentam $\mathrm{R}^{2}$ maiores que 0,50 . Conforme a classificação dada por AZEVEDO (2004), esses resultados mostram, em geral, ajustes dos semivariogramas que possibilitam a obtenção dos mapas da distribuição espacial dos atributos de forma confiável.

Na Figura 4, observam-se os mapas de isolinhas da distribuição espacial dos valores de VTP e MiP nas duas áreas estudadas, construídos por krigagem. O VTP apresenta valores maiores na parte superior da PA e melhor distribuição espacial na área de VN. A região de menores valores de MiP na PA coincide com os menores valores de VTP. A distribuição espacial da VTP e MiP na área de PA tem o mesmo comportamento verificado por SOUZA et al. (2004) em solo com elevado tráfego de máquinas, na cultura de cana-de-açúcar. 

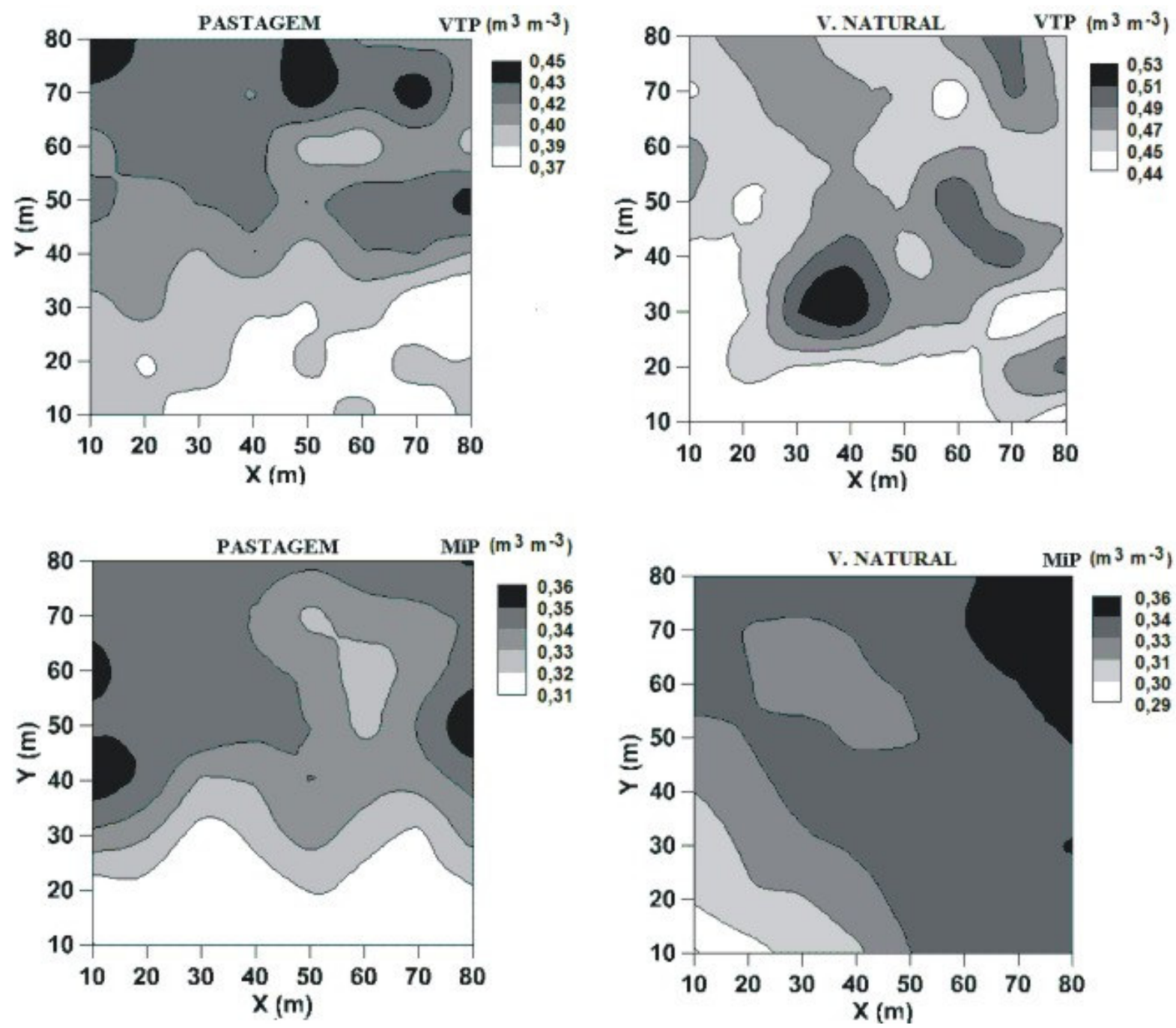

FIGURA 4. Mapas de isolinhas dos atributos do solo, volume total de poros - VTP $\left(\mathrm{m}^{3} \mathrm{~m}^{-3}\right)$ e microporosidade - MiP $\left(\mathrm{m}^{3} \mathrm{~m}^{-3}\right)$ sob pastagem (PA) e vegetação secundária em regeneração natural (VN). Isoline maps of soil attributes total volume of pores VTP $\left(\mathrm{m}^{3} \mathrm{~m}^{-3}\right)$ and microporosity - MiP $\left(\mathrm{m}^{3} \mathrm{~m}^{-3}\right)$ under pasture (PA) and secondary vegetation in natural regeneration $(\mathrm{VN})$.

Os mapas de Ds (Figura 5) mostram a localização dos maiores valores na região inferior das duas áreas estudadas, região de menor VTP e MiP. Esse fato indica preferência dos animais em se acumularem nessa região, consequentemente, maiores cargas são aplicadas ao solo pelo pisoteio e, cabe lembrar, a VN foi utilizada no passado como área de pastagem. A distribuição espacial da Ds tem o comportamento inverso à do VTP, porém com o mesmo padrão espacial apresentado pelos semivariogramas escalonados.

Na Figura 6, a RP apresentou comportamento esperado, com valores maiores na parte central e inferior da área de VN, justamente na região de menor VTP. Esses resultados estão distribuídos espacialmente aos valores de Ds, com concentração de maiores valores a partir da parte central para a baixa do terreno. Segundo KLEIN et al. (1998), a RP apresenta estreita relação com a Ds, embora se deva reconhecer, como afirmam TORRES \& SARAIVA (1999), que a RP pode ser mais afetada pela U no momento da amostragem do que pela Ds. BELTRAME et al. (1981) afirmam que, com a redução do teor de água no solo, ocorre aumento na RP decorrente da maior coesão entre partículas, o que visualmente não ficou evidenciado neste estudo. 

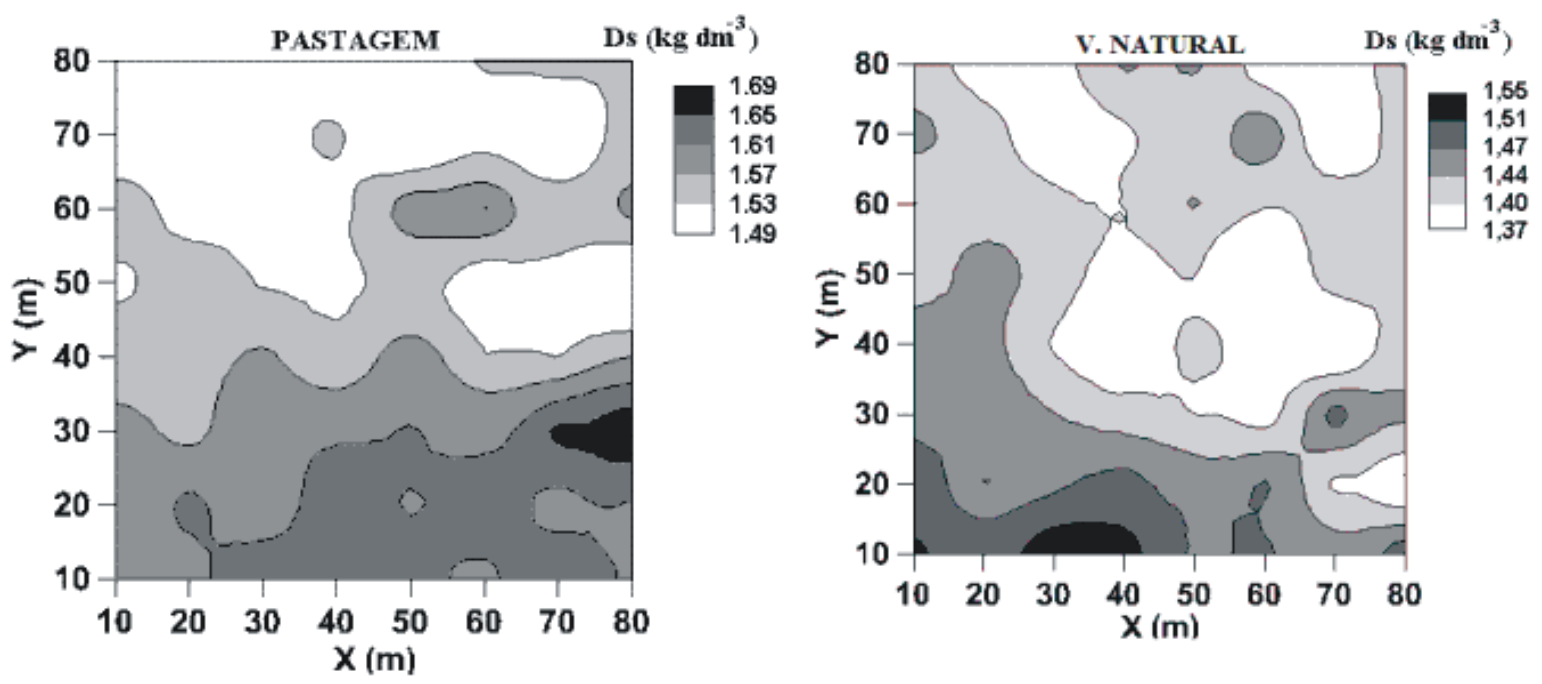

FIGURA 5. Mapas de isolinhas do atributo densidade do solo - Ds $\left(\mathrm{kg} \mathrm{dm}^{-3}\right)$ sob pastagem (PA) e vegetação secundária em regeneração natural (VN). Isoline maps of the attribute bulk density - Ds $\left(\mathrm{kg} \mathrm{dm}^{-3}\right)$ under pasture (PA) and secondary vegetation in natural regeneration $(\mathrm{VN})$.
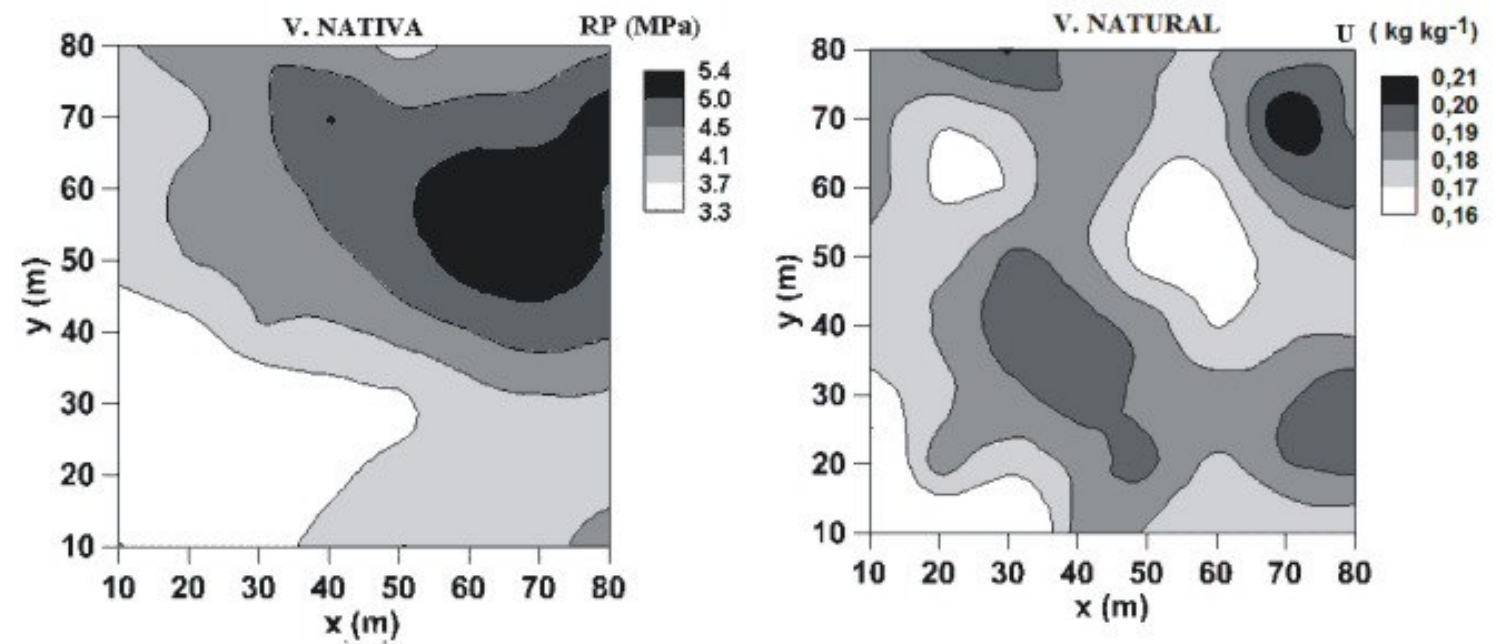

FIGURA 6. Mapas de isolinhas para os atributos resistência do solo à penetração - RP (MPa) e umidade do solo - $U\left(\mathrm{~kg} \mathrm{~kg}^{-1}\right)$ sob vegetação secundária em regeneração natural. Isoline maps for the soil attributes resistance to the penetration - RP (MPa) and soil moisture - $U\left(\mathrm{~kg} \mathrm{~kg}^{-1}\right)$, under secondary vegetation in natural regeneration (VN).

\section{CONCLUSÕES}

O volume total de poros, a microporosidade, a densidade do solo e a umidade, nas duas áreas, apresentam baixa variabilidade indicada pelo coeficiente de variação.

Os atributos físicos do solo mostram estrutura de dependência espacial de moderada a forte, exceto para a macroporosidade e a resistência do solo à penetração.

O volume total de poros e a densidade do solo apresentam o mesmo padrão espacial na área sob pastagem. 

físicos.

O solo sob as duas formas de uso apresenta estrutura espacial diferenciada para os atributos

\section{REFERÊNCIAS}

ALBUQUERQUE, J.A.; SANGOI, L.; ENDER, M. Efeitos da integração lavoura-pecuária nas propriedades físicas do solo e características da cultura do milho. Revista Brasileira de Ciência do Solo, Campinas, v.25, n.3, p.717-723, 2001.

ALVARENGA, R.C.; COSTA, L.M.; MORA FILHO, W.; REAGAZZI, A.J. Crescimento de raízes de leguminosas em camadas de solo compactadas artificialmente. Revista Brasileira de Ciência do Solo, Campinas, v.20, n.2, p.319-326, 1996.

ARCHER, J.R.; SMITH, P.D. The relation between bulk density available water capacity, and air capacity of soils. Journal of Soil Science, London, v.23, n.4, p.475-480, 1972.

AZEVEDO, E. C. Uso da geoestatística e de recursos de geoprocessamento no diagnóstico da degradação de um solo argiloso sob pastagem no Estado de Mato Grosso. 2004. 132 f. Tese (Doutorado em Água e Solo) - Universidade Estadual de Campinas, Campinas, 2004.

BELTRAME, L.F.S.; GONDIM, L.A.P.; TAYLOR, J.C. Estrutura e compactação na permeabilidade de solos do Rio Grande do Sul. Revista Brasileira de Ciência do Solo, Campinas, v.5, n.1, p.145-149, 1981.

CASTRIGNANO, A.; GIUGLIARINI, L.; RISALITI, R.L. Study of spatial relationships among soil physico-chemical properties of a field in central Italy using multivariate geostatistics.

Geoderma, Amsterdam, v.97, n.1/2, p.39-60, 2000.

EMBRAPA. EMPRESA BRASILEIRA DE PESQUISA AGROPECUÁRIA. Manual de métodos de análise de solo. Rio de Janeiro, 1997. 212 p.

EMBRAPA. EMPRESA BRASILEIRA DE PESQUISA AGROPECUÁRIA. Sistema brasileiro de classificação do solo. Brasília, 1999. $412 \mathrm{p}$.

GUIMARÃES, E.C. Variabilidade espacial de atributos de um Latossolo Vermelho-Escuro textura argilosa da região do cerrado, submetido ao plantio direto e ao plantio convencional. $2000.92 \mathrm{f}$.

Tese (Doutorado em Engenharia Agrícola) - Universidade Estadual de Campinas, Campinas, 2000.

IMHOFF, S.; SILVA, A.P.; DIAS JÚNIOR, M.S.; TORMENA, C.A. Quantificação de pressões críticas para o crescimento das plantas. Revista Brasileira de Ciência do Solo, Campinas, v.25, n.1, p.11-18, 2001.

LANI, J. L. Estratificação de ambientes na Bacia do Rio Itapemirim, no sul do Estado do Espírito Santo. 1987. Dissertação (Mestrado em Solos) - Universidade Federal de Viçosa, Viçosa - MG, 1987.

JORGE, J.A. Física e manejo de solos tropicais. Campinas: Instituto Campineiro de Ensino Agrícola, 1985. 328 p.

KLEIN, V.A.; LIBARDI, P.L.; SILVA, A.P. Resistência mecânica do solo à penetração sob diferentes condições de densidade e teor de água. Engenharia Agrícola, Jaboticabal, v.18, n.1, p.4554, 1998.

MAGALHÃES, R.T. de; KLIEMANN, H. J. de; OLIVEIRA, I.P. de. Evolução das propriedades físicas de solos submetidos ao manejo do sistema barreirão. Pesquisa Agropecuária Tropical, Brasília, v.31, n.1, p.7-13, 2001.

OLIVEIRA, R.B. Mapeamento e correlação de atributos do solo e de plantas de café conilon para fins de agricultura de precisão. 2007. 129 f. Dissertação (Mestrado em Produção Vegetal) Universidade Federal do Espírito Santo, Alegre, 2007. 
ROBERTSON, G.P. GS $S^{+}$: Geostatistics for the environmental sciences - GS ${ }^{+}$User's Guide. Plainwell: Gamma Design Software, 1998. 152 p.

SATTLER, M.A. Variabilidade espacial de atributos de um Argissolo Vermelho-Amarelo sob pastagem e vegetação nativa na bacia hidrográfica do Itapemirim. 2006. 80 f. Dissertação (Mestrado em Produção Vegetal) - Universidade Federal do Espírito Santo, Alegre, 2006.

SÁ, R.C.; PEREIRA, M.G.; FONTANA, A. Características físicas e químicas de solos de tabuleiros em Sooretama (ES). Floresta e Ambiente, Rio de Janeiro, v.10, n.2, p.95 - 99, 2003.

SILVA, F.M.; SOUZA, Z.M.; FIGUEIREDO, C.A.P.; MARQUES JÚNIOR, J.; MACHADO, R.V. Variabilidade espacial de atributos químicos e de produtividade na cultura do café. Ciência Rural, Santa Maria, v.37, n.2, p.401-407, 2007.

SOUZA, Z.M.; ALVES, M.C. Propriedades físicas e teor de matéria orgânica em um Latossolo Vermelho de cerrado sob diferentes usos e manejos. Acta Scientiarum: Agronomy, Maringá, v.25, n.1, p.27-34, 2003.

SOUZA, Z.M.; MARQUES JÚNIOR, J.; PEREIRA, G.T.; BENTO, M.J.C. Variabilidade espacial de atributos físicos de um Latossolo Vermelho sob cultivo de cana-de-açúcar. Revista Brasileira de Engenharia Agrícola e Ambiental, Campina Grande, v.8, n.1, p.51-58, 2004.

SPERA, S.T. Inter-relações entre propriedades físico-hídricas do solo e a ocorrência de vegetação de mata e campo adjacentes no Alto Rio Grande - MG. 1995. 78 f. Dissertação (Mestrado em Ciência do Solo) - Universidade Federal de Lavras, Lavras, 1995.

STOLF, R. Teoria e teste experimental de fórmulas de transformação dos dados de penetrômetro de impacto em resistência do solo. Revista Brasileira de Ciência do Solo, Campinas, v.15, n.3, p.229235, 1991.

TORMENA, C.A. A compactação do solo em agroecossistemas agrícolas. In: REUNIÃO BRASILEIRA DE MANEJO E CONSERVAÇÃ̃ DO SOLO E DA ÁGUA, 14., 2002, Viçosa. Anais... Viçosa: Sociedade Brasileira de Ciência do Solo, 2002 . 4 p. 1 CD-ROM).

TORRES, E.; SARAIVA, O.F. Camadas de impedimento do solo em sistemas agrícolas com a soja. Londrina: Empresa Brasileira de Pesquisa Agropecuária, 1999. 58 p. (Circular Técnica, 23).

VIEIRA, S.R.; HATFIELD, T.L.; NIELSEN, D.R. Geoestatiscal theory and application to variability of some agronomical properties. Hilgardia, Oakland, v.51, n.1, p.1- 75, 1983.

VIEIRA, S.R. Geoestatística em estudos de variabilidade espacial do solo. In. NOVAES, R. F.; ALVAREZ V.V.H.; SCHAEFER, C.E.G.R. Tópicos em ciência do solo. Viçosa: Sociedade Brasileira de Ciência do Solo, 2000. v.1, p. 2-54.

WARRICK, A.W.; NIELSEN, D. R. Spatial variability of soil physical properties in the field. In: HILLEL, D. (Ed.). Application of soil physics. New York: Academic Press, 1980. p.319-324.

WEBSTER, R. Quantitative spatial analysis of soil in the field. Advances in Soil Science, New York, v.3, n.1, p.1-70, 1985.

ZIMBACK, C.R.L. Análise espacial de atributos químicos de solos para fins de mapeamento da fertilidade. 2001. 114 f. Tese (Livre-Docência em Levantamento do Solo e Fotopedologia) Faculdade de Ciências Agronômicas, Universidade Estadual Paulista, Botucatu, 2001. 